\title{
VALIDATION OF A MEDIA SELECTION FRAMEWORK THROUGH PRACTICAL USE
}

\author{
Chigozie Onyekaba, Russell Campion, Anthony Atkins \\ Staffordshire University (UNITED KINGDOM)
}

\begin{abstract}
Appropriate use of multimedia brings potential education and learning benefits to students. It is widely accepted that people learn better when the full potentials of multimedia are put to correct use, during the design of educational / learning material. In a previous paper by the authors, we presented an initial media selection framework, used for mapping multimedia to educational concepts. In this paper, we present results of experiments carried out using the framework practically for educational design. Specifically, the paper focuses on validating the framework, in essence, assessing the extent to which it improves learning. The validation experiment compared the performance of students that learned from material designed using the framework, with the performance of students that learned from existing learning materials, both sets of material contained similar educational concepts. We also performed reliability tests (assessing whether the framework produces the same media output for the same educational concept) and usability tests (assessing whether users found the framework easy to use). This paper presents and discusses the results of these tests.
\end{abstract}

Keywords: Multimedia, media selection, educational material.

\section{INTRODUCTION}

The use of multimedia in education is known to have a positive effect on learning as shown by numerous studies ([1], [2], [3], [4]). This is known as the multimedia effect. As a result of effects of multimedia on learning, a number of theories about the use of multimedia have emerged. Such theories include the modality theory ([3], [5], [6]), the multimedia principle, the coherence principle, contiguity principle, etc. [1]. Although there is a considerable amount of information currently known about multimedia and its effect on the learning process, not much had been known about how to methodically select appropriate media types for presenting educational concepts. Due to this, educational material developers tend to rely on intuition and personal experience when developing learning materials. While reliance on intuition and personal experience may be acceptable in some cases, it could potentially be problematic especially when the learning content is being created by inexperienced educational materials developers. The importance of having a proven methodical approach cannot be overstated because inappropriate use of multimedia in educational materials can have negative effects on the learning process. Guidelines for media selection have been created in the past, such as the multimedia taxonomy framework [7], the multimedia advisor tool [8], etc, but some of these guidelines do not take into account the vast amount of media types (and sub-types) currently available and some are more suited to the design of general interfaces, rather than being specifically designed for educational materials. This formed the basis of our previous work [9], which involved the development of a method/approach for selecting appropriate media types to present educational information. In the next section, we would briefly describe this media selection framework.

\section{MEDIA SELECTION FRAMEWORK}

In our previous paper, we described a media taxonomy and a media selection framework [9]. The media taxonomy breaks down main media types into subtypes based on their information presenting attributes or capabilities. The media selection framework we developed, makes use of this taxonomy in outputting a media type for use in educational materials. Figure 1 is a hierarchical diagram of the media taxonomy we developed. In order to select relevant media types for presenting educational concepts, we produced a set of information identifiers and mapped these information identifiers to suitable media types. These media types were arranged in order of appropriateness. An educational material designer would normally perform task analysis and then for each information presentation task, the designer would go through the list of identifiers to select the identifiers that are relevant to the information presentation tasks. Once a set of identifiers are selected, the designer would collate all the media types that map to the selected identifiers and the most occurring media type would be the 
suitable media type for the information presentation task. In situations where there are multimodal media type outputs, the designer would select the media type that occurs first, from the list of media types that map to the selected identifiers. In some cases, there may be a second identifier selection stage before a media type is outputted, however the same process is typically followed. We built a web based tool to simplify this process and we demonstrated how this framework can be applied to media selection, in our previous paper [9]. In the next section, we discuss a set of experiments to assess and validate this framework.

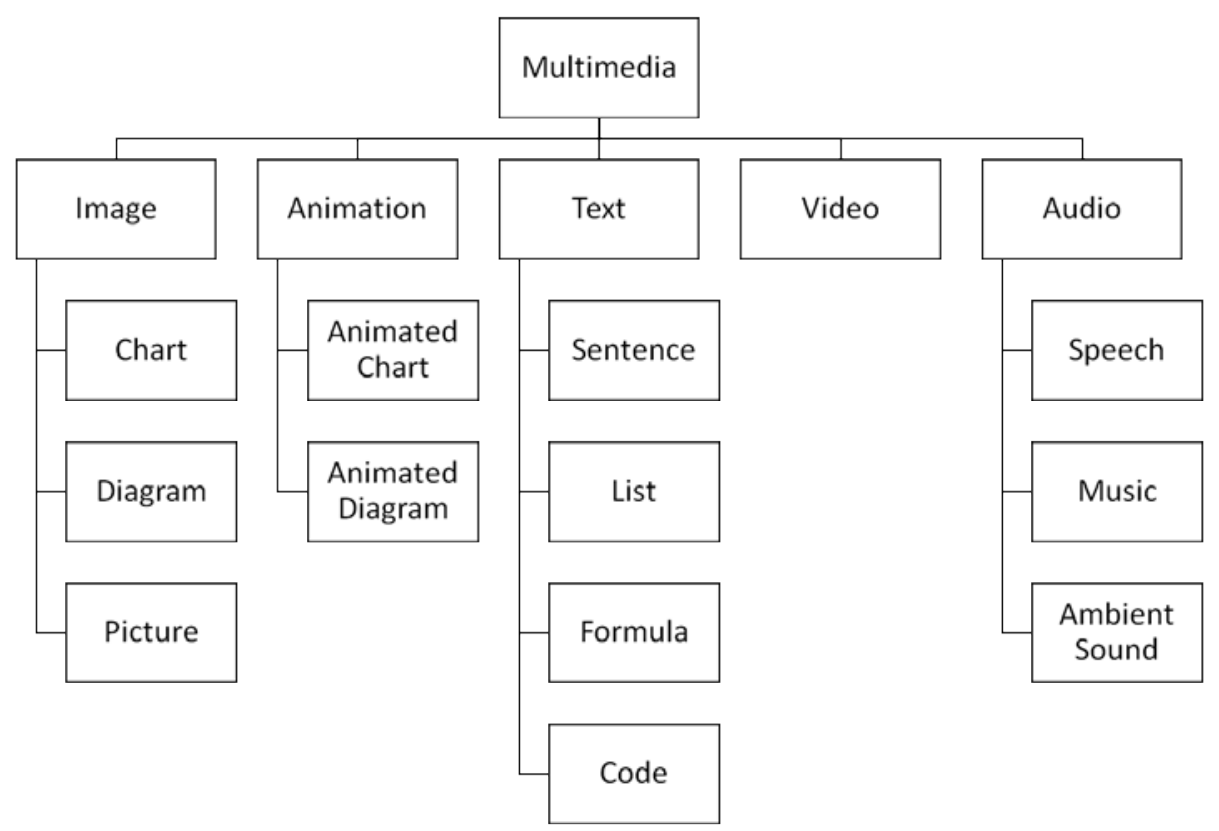

Figure 1: Media Taxonomy Hierarchical Diagram

\section{EXPERIMENTS}

In this section, we outline the procedure for the two experiments undertaken in this study. The first experiment conducted was the validity experiment, while the second experiment was the reliability and usability experiment. The aim of the first experiment was to validate the media selection framework we developed. In essence, is there a statistically significant improvement in learning when the media selection framework is used to develop learning materials, compared to similar learning materials? The aim of the second experiment was to assess the usability and reliability of the media selection framework. In essence, do users find it easy to learn, useful and usable? Does it output the media type intended by the authors when used for the same information presentation task?

\subsection{Validity Experiment}

The validity experiment aimed to answer a very important question. Does the media selection framework improve learning when used to design learning materials? In order to test for improvements in learning, we would present two groups of learners with two sets of learning materials (one for each group). One would be an existing material and the other would be a modified version of the initial material. The modification of the existing learning material would be done using the framework guidelines. The participants would then be asked to undertake learning tests. Higher scores in learning tests for participants using the modified material (compared to the other participants using the existing material) would mean that learning was improved.

\subsubsection{The Design}

An independent design was used for the validation experiment. There were two groups involved in this experiment, a control group and an experimental group. The learning material used for this experiment was obtained from khanacademy.org. This learning material (obtained on khanacademy.org) was used for the control group. The learning material was then modified, using the media selection framework, to produce a learning material which essentially contained the same information but presented some of the information in a slightly different way, using different media types where 
applicable, in accordance with the media selection framework. The modifications required were not major as the original material was fairly in compliance with some of the guidelines of the media selection framework.

\subsubsection{The Participants}

Participants were recruited and split into two different groups of roughly similar characteristics. There were sixteen participants per group, consisting of eight males and eight females. The participants were university students, mostly from the School of Computing in Staffordshire University.

\subsubsection{The Procedure}

Participants were asked to study the learning materials for their group and were afterwards asked to complete a multiple choice test containing ten questions, aimed at assessing learning retention. The learning activity had to be completed within twenty minutes and any participant exceeding that time would have to be stopped. The retention test had to be completed within ten minutes and participants would be stopped if they exceeded that time limit.

\subsection{Reliability and Usability Experiment}

This part of the study aimed to assess the reliability of the media selection framework in outputting the media types intended by the authors. The experiment also aims to assess the usability of the framework, in essence, to answer questions such as, is the framework easy to use, do users find the framework easy to learn, useful, etc.

\subsubsection{The Design}

For the reliability and usability experiments, the participants would be taught how to use the framework to select media to represent information. A web interface/tool was also developed to simplify the media selection task of the framework. The interface would be made available to the participants and they would be taught how to use it. After learning to use the framework, they would be asked to select appropriate media types for various educational scenarios and then asked questions about the ease of use, ease of learning and usefulness of the framework.

\subsubsection{The Participants}

Seven participants (three females and four males) were recruited for this experiment. All the participants had at least one year experience in teaching and designing learning materials, the most having six years of teaching and design experience. The mean experience in years was 2.57 .

\subsubsection{The Procedure}

A PowerPoint slideshow was used to train the participants on how to use the media selection framework. Participants were also taught how to use the web tool for the media selection task. After the training activity, participants were presented with ten educational scenarios and were asked to use the media selection web tool to identify a suitable media type for each of the educational scenarios. At the end of the media selection task, participants were asked to complete a scaled down version of Lund's USE questionnaire [10]. Finally, a NASA TLX workload assessment [11] was done to measure the workload of the media selection task.

\section{RESULTS}

In this section, we discuss the analysis of the data obtained from both experiments.

\subsection{Validity Test results}

An independent samples t-test statistic was used to analyse the results of the validity experiment. The retention test scores of the control group was compared with the retention test scores of the experimental group. At first, the data were checked for normality using Shapiro-Wilks test and both groups satisfied the assumption of normality. Figures 2 and 3 show a normal $Q-Q$ plot of the test scores for participants in the control group and the experimental group. 


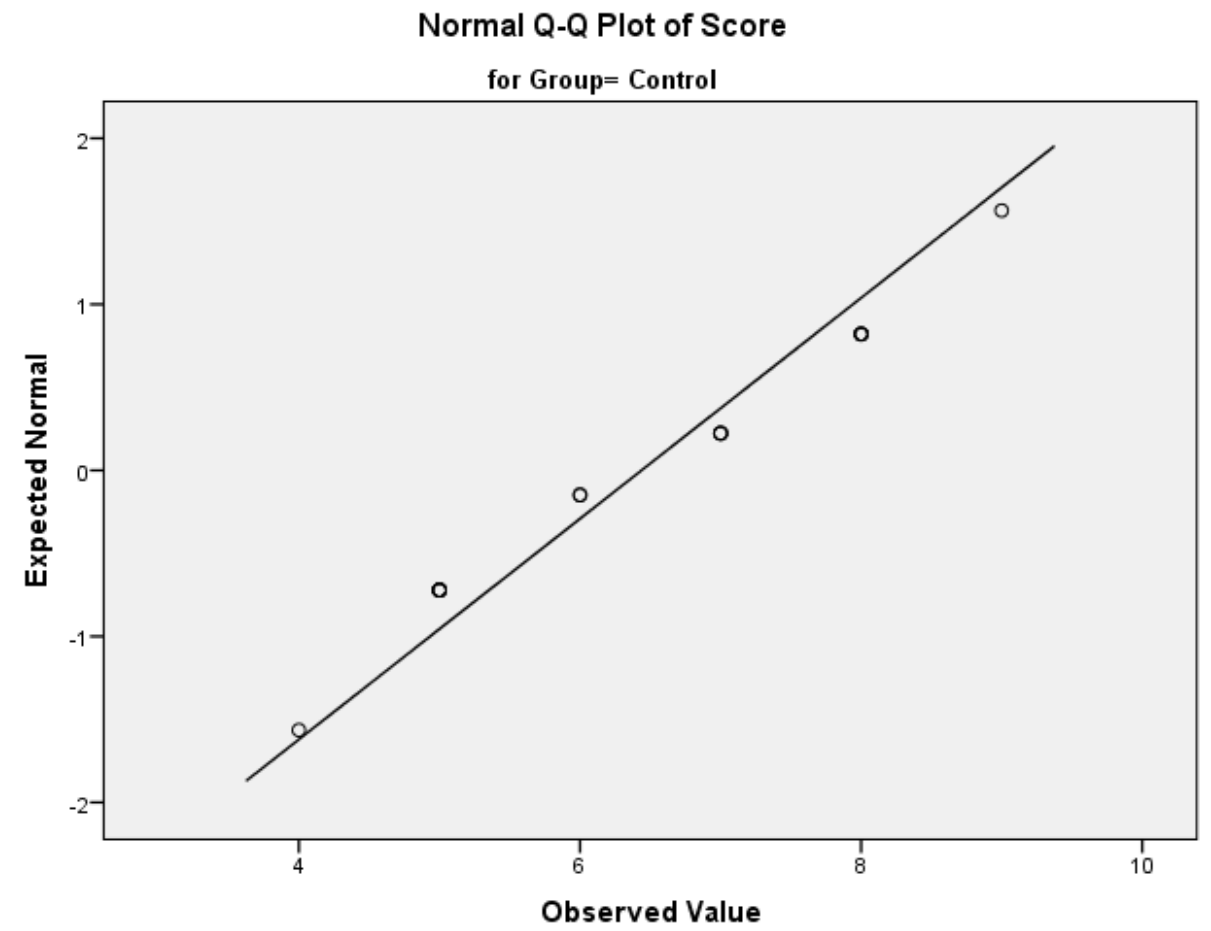

Figure 2: Normality Plot - Control Group

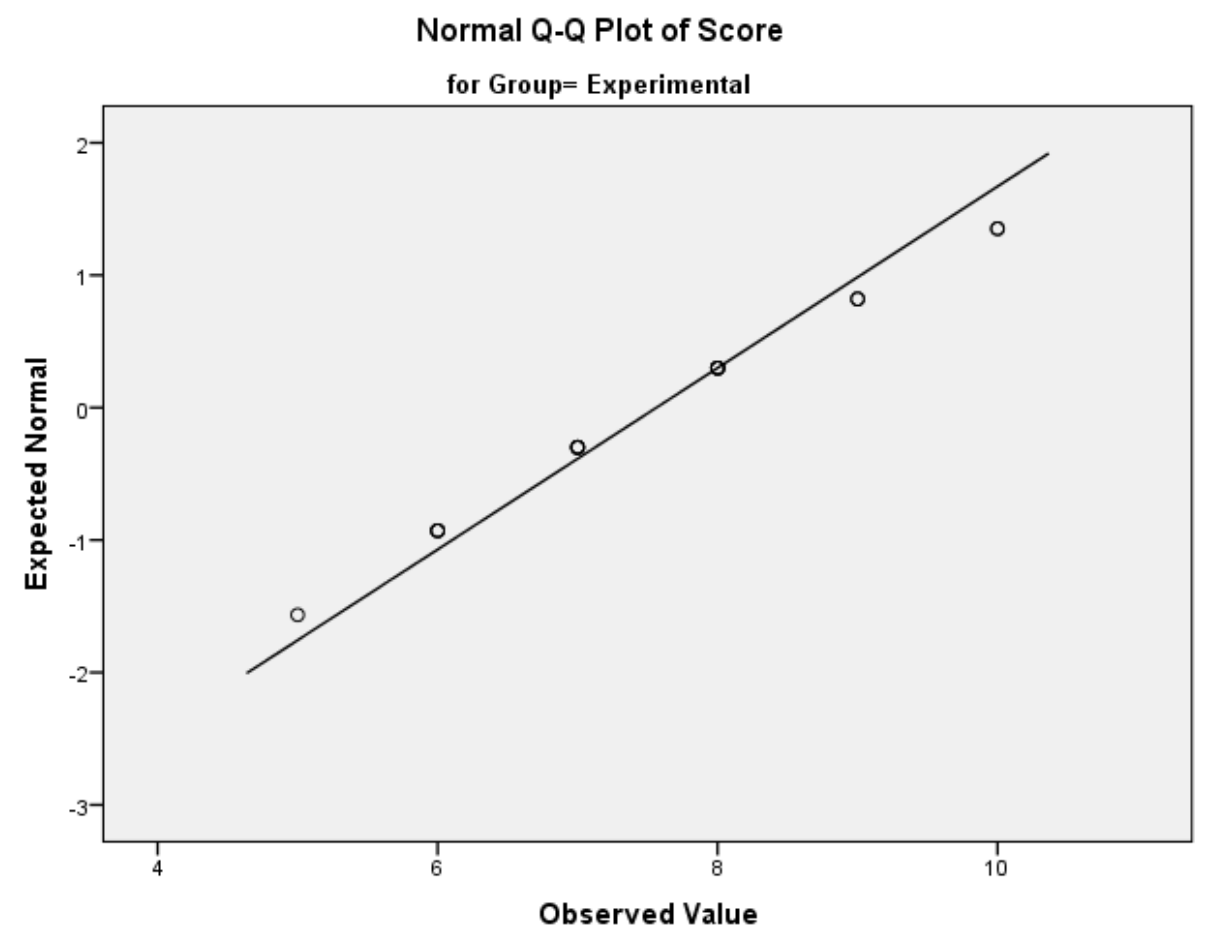

Figure 3: Normality Plot - Experimental Group

Participants in the control group got a mean test score of 6.44 (SD = 1.504). In comparison, participants in the experimental group got a numerically larger mean test score of $7.56(S D=1.459)$. To test whether the higher learning retention test scores of the experimental group, compared to the control group, was statistically significantly, an independent samples t-test was done. The assumption of homogeneity of variances was tested and satisfied via Levene's test, $F(30)=0.238, p=0.629$. The results of the independent samples t-test showed that the higher mean score of experimental group, 
compared to the control group, was statistically significant, $t(30)=2.147, p=0.04$. Cohen's $d$ was estimated at 0.76 , which is a moderate effect size, based on Cohen's guidelines [12].

\subsection{Reliability and Usability Test Results}

In the media selection task, a media output that matched the media type intended by the developers of the media selection framework, was regarded as a successful media selection while an incorrect media selection output was regarded as an unsuccessful media selection. The performance scores of the seven participants (out of the ten media selection tasks) ranged between 4 and $9(M=5.43, S D=$ 1.90). The overall workload score of the subjective workload assessment of the media selection task ranged from 32 to 66 with an average of 50 .

The usability questionnaire made use of a seven point Likert scale ranging from strongly disagree (1) to strongly agree (7). Table 1 shows the frequency distribution (in percentage) of the USE questionnaire data. The positive column represents ratings from 5 - 7 (those that tended to agree, agreed or strongly agreed with the statement), the neutral column represents ratings for 4 (those with a neutral opinion about the statement), while the negative column represents ratings for $1-3$ (those that tended to disagree, disagreed or strongly disagreed with the statement).

Table 1: Frequency distribution of the USE questionnaire data

\begin{tabular}{|l|c|c|c|}
\hline & POSITIVE & NEUTRAL & NEGATIVE \\
\hline USEFULNESS & & & \\
\hline It helps me be more effective & $71.43 \%$ & $14.29 \%$ & $14.29 \%$ \\
\hline It helps me to be more productive & $71.43 \%$ & $14.29 \%$ & $14.29 \%$ \\
\hline It is useful & $85.71 \%$ & $0.00 \%$ & $14.29 \%$ \\
\hline EASE OF USE & & & \\
\hline It is easy to use & $57.14 \%$ & $14.29 \%$ & $28.57 \%$ \\
\hline It is user friendly & $57.14 \%$ & $14.29 \%$ & $28.57 \%$ \\
\hline Using it is effortless & $42.86 \%$ & $42.86 \%$ & $14.29 \%$ \\
\hline EASE OF LEARNING & & & \\
\hline I learned to use it quickly & $57.14 \%$ & $0.00 \%$ & $42.86 \%$ \\
\hline I easily remember how to use it & $71.43 \%$ & $14.29 \%$ & $14.29 \%$ \\
\hline It is easy to learn to use it & $57.14 \%$ & $14.29 \%$ & $28.57 \%$ \\
\hline SATISFACTION & & & \\
\hline I am satisfied with it & $57.14 \%$ & $14.29 \%$ & $28.57 \%$ \\
\hline I would recommend it to a friend & $71.43 \%$ & $0.00 \%$ & $28.57 \%$ \\
\hline I feel I need to have it & $57.14 \%$ & $14.29 \%$ & $28.57 \%$ \\
\hline
\end{tabular}

\section{DISCUSSION AND CONCLUSION}

Multimedia has been known to hold huge potentials for learners. The promise of multimedia is that people learn and retain information better when multimedia is put to good use in learning and the results of our study have further confirmed this principle. However, in the absence of a methodical approach for selecting media for educational materials, we developed a framework for educational media selection and embedded this into a simple-to-use web application. The results of the validity experiment in this paper have shown that the framework we developed has a real potential to improve learning when appropriately used. Statistically significant differences were observed between those who learned using educational material that employed our framework in its development and those who learned using the existing learning material. While the results of the validity experiment showed that using the framework did improve learning, the results of the usability assessment showed that 
more work may be required to make it easier for users to learn to use the framework because the consequence of users not fully understanding how to use the framework is that incorrect media types may be used for educational material design, thereby negatively affecting learning. In Table 1, we can see that users thought the framework helped them become more effective $(71.43 \%)$, more productive $(71.43 \%)$ and users considered the framework useful $(85.71 \%)$. However, moving on to its ease of use and ease of learning, the data shows a drop in positive feedback received. The reduced ease of learning was also evident in the reliability experiment as participants were, on average, only able to correctly answer just over 5 of the media selection task (the average score was 5.43 out of 10). Some of the feedback we received during the second experiment was that it would be very helpful to expand the descriptions of the information identifiers and also to add more examples covering different scenarios when the identifier would be selected. Another feedback we received was that rather than outputting just one media type, we could (where there may be other reasonably suitable media types) consider outputting one media type as the main recommended media type to use and perhaps one or two others as possible media types that could be used to present the information. Work is now being done to improve the ease of learning of the framework and also to incorporate some of these recommendations to the framework.

\section{REFERENCES}

[1] Clark, Ruth C., and Richard E. Mayer (2011). E-learning and the science of instruction: Proven guidelines for consumers and designers of multimedia learning. John Wiley \& Sons, 2011.

[2] Najjar, L. (1998). Principles of educational multimedia user interface design. Human Factors: The Journal of the Human Factors and Ergonomics Society, 40(2), pp.311-323..

[3] Mayer, R. (2001). Multimedia learning. New York: Cambridge University Press.

[4] Mayer, R. (2003). The promise of multimedia learning: using the same instructional design methods across different media. Learning and instruction, 13(2), pp.125-139.

[5] Moreno, R. and Mayer, R. (1999). Cognitive principles of multimedia learning. Journal of Educational Psychology, 91, pp.358-368.

[6] Low, R. and Sweller, J. (2005). The modality principle in multimedia learning. The Cambridge handbook of multimedia learning, 147, pp.158.

[7] Heller, R. S., Martin, C. D., Haneef, N., and Gievska-Krliu, S. (2001). Using a theoretical multimedia taxonomy framework. Journal on Educational Resources in Computing (JERIC)

[8] Sutcliffe, A. G., Kurniawan, S., and Shin, J. E. (2006). A method and advisor tool for multimedia user interface design. International Journal of Human-Computer Studies, 64(4), 375-392.

[9] Onyekaba C., Campion R., Atkins A. (2016). A framework for mapping multimedia to educational concepts. INTED2016 Proceedings, pp. 7987-7996.

[10] Lund A. (2001). Measuring Usability with the USE Questionnaire.

[11] Hart, S. (2006). October. NASA-task load index (NASA-TLX): 20 years later. In Proceedings of the human factors and ergonomics society annual meeting. pp. 904-908.

[12] Cohen, J. (1992). A power primer. Psychological bulletin, 112(1), pp.155. 